\title{
Region of Attraction Analysis via Invariant Sets
}

\author{
Giorgio Valmorbida
}

\author{
James Anderson
}

\begin{abstract}
In this work we address the problem of estimating the region of attraction (RA) of a nonlinear dynamical system. We propose a method that uses a Lyapunov type approach to obtain an estimate of the RA, however, the obtained invariant sets are not level sets of the Lyapunov function certificates. We then restrict our attention to systems governed by polynomial vector fields and semi-algebraic sets and provide an algorithm that with each iteration is guaranteed to enlarge the estimate of the RA.
\end{abstract}

\section{INTRODUCTION}

The problem of computing the region of attraction (RA) of asymptotically stable equilibria or inner estimates to this set (ERA) has been historically motivated by the recovery of operating points in power systems. However its relevance is immediately clear for many practical nonlinear system for which we can only guarantee local properties of operating points.

With a converse Lyapunov theorem [2, Theorem 19], Zubov answered the question [2, p.3] "[...] Is it possible, with the help of the Lyapunov function to find a region of variation of the initial values $x_{0}$ such that $\left\|\phi\left(t, x_{0}\right)\right\| \rightarrow 0$ as $t \rightarrow \infty$ ?'. The theorem states that if $S$ is the RA of an equilibrium then the existence of a Lyapunov function (LF) satisfying some conditions on such a set $S$ is necessary and sufficient. However, computing the actual RA following Zubov's theorem requires the solution of a partial differential equation which may be difficult to obtain. The main obstacle being to satisfy the semidefiniteness of the Lyapunov function derivative in the whole set of converging trajectories. However it is not difficult to obtain semidefinite functions that satisfy the proposed conditions locally, providing only ERAs for the equilibrium point of interest. In fact, an approximate solution obtained with a series expansion of the LF was proposed [2, p.91] and is now referred to as Zubov's Method.

In [3] Zubov's theorem is presented in an alternative form using Lyapunov functions mapping $\mathbb{R}^{n}$ to $\mathbb{R}_{\geq 0}$. One of the conditions in [3] imposes that the LF $V(x)$ satisfy $V(x) \rightarrow \infty$ whenever $x \rightarrow \partial S$ or

\footnotetext{
Department of Engineering Science, University of Oxford, 17 Parks Road, OX1 3PJ Oxford, United Kingdom, \{giorgio.valmorbida, james. anderson\} deng.ox.ac.uk. J. Anderson is also affiliated to St. John's College, University of Oxford, Oxford, U.K. G. Valmorbida is also affiliated to Somerville College, University of Oxford, Oxford, U.K. Work partly supported in part by EPSRC grant EP/J010537/1.
}

$|x| \quad \rightarrow \quad \infty$, these functions were called maximal Lyapunov functions. This is a concept distinct from maximal Lyapunov set, which is currently used [4] to define the largest level set of a LF contained in the RA. The computing the latter is of course a problem of interest since one might want to know the best estimate that it is possible to get with a given Lyapunov function.

In recent years sufficient conditions for local stability analysis, requiring invariance and contractiveness of a set led to numerical methods for the estimation of the RA with different classes of polynomial Lyapunov functions. The methods presented in [5]-[7] compute Lyapunov functions and obtain an ERA given by the level set of these functions. These methods rely on the solution of non-convex constraints given by polynomial inequalities derived with the Positivstellensatz [8, Theorem 2.14] and require a coordinatewise search since some polynomial variables appear multiplying the Lyapunov function. For the case of a given LF, the computation of the maximal Lyapunov set was pursued in [9]. For a detailed description of sum of squares methods for RA estimation the reader is referred to [10]. In [11] the theory of moments is used to estimate the RA of uncertain polynomial systems. We also find in the literature numerical methods exploiting topological properties of the boundary of the RA which led authors to obtain estimates computing trajectories and computing the whole set of equilibria of the system. However the complexity of such methods has restricted them to only 2-dimensional examples [12]. Recently in [1] set advection methods are described.

There is, however, conservativeness of imposing the level sets of the Lyapunov function to be the ERA as observed in [13, p.320] "Estimating the region of attraction by $\Omega_{c}=\{x \mid V(x) \leq c\}$ is simple but usually conservative. According to LaSalles's theorem ... we can work with any compact set $\Omega \subset D$ provided we can show that $\Omega$ is positively invariant." This suggests that one could look for sets which are not contractive but only invariant.

In this paper we derive conditions based on Lyapunov stability results that guarantee the convergence of trajectories in an invariant region to a level set of the LF which is contractive and invariant. Therefore guaranteeing such an invariant set to be an ERA. We then propose a numerical method that applies to poly- 
nomial systems, the computed ERAs are described by semialgebraic sets obtained from the solution to sum of squares programs.

The paper is organized as follows: in Section II and Section III we recall some definitions and review the structure of existing algorithms for ERA computation. Section IV presents the conditions for an invariant region to be the ERA of the origin. These conditions are the base for an algorithm presented in SectionIV-B allowing for the numerical solution through a sequence of sum-of-squares programs. Section V illustrates the application of the algorithm and, finally, in Section VI we review the results and discuss the their connections to the class of rational Lyapunov functions.

\section{PRELIMINARIES}

Let $\mathbb{R}, \mathbb{R}_{\geq 0}, \mathbb{R}_{>0}$ and $\mathbb{R}^{n}$ denote the field of reals, non-negative reals, positive reals and the $n$ dimensional Euclidean space respectively. The function $f: \mathbb{R}^{n} \rightarrow \mathbb{R}$ is said to be positive definite if $f(x)>0$ for all non-zero $x \in \mathbb{R}^{n}$, similarly if $f(x) \geq 0$ for all $x \in \mathbb{R}^{n}$ then $f$ is said to be positive semidefinite. $c o(\mathcal{X})$ denotes the convex hull of the set $\mathcal{X}$ and $\partial \mathcal{X}$ its boundary. For $x \in \mathbb{R}^{m}$ the ring of polynomials in $m$ variables is denoted by $\mathbb{R}\left[x_{1}, \ldots, x_{m}\right]$, when it is clear from context we will use $\mathbb{R}[x]$. A polynomial, $p(x)$, is said to be a sum of squares (a subset of the polynomial ring) if there exists a finite set of polynomials $g_{1}(x), \ldots, g_{p}(x)$ such that $p(x)=\sum_{i=1}^{p} g_{i}^{2}(x)$. The set of sum-of-squares, (SOS), polynomials in $x$ is denoted by $\Sigma\left[x_{1}, \ldots, x_{m}\right]$ which can be abbreviated to $\Sigma[x]$. Equivalently $p(x)$ is SOS if there exists a positive semidefinite matrix $Q$ such that $p(x)=Z^{T}(x) Q Z(x)$ where $Z(x)$ is a given vector of monomials. Note that the search for $Q$ can be formulated as a semidefinite program and thus solved using convex optimization techniques [14]. It may be necessary at times to specify the number of variables and a degree bound on a (SOS) polynomial, in which case we use the notation $\left(\Sigma_{m, d}[x]\right) \mathbb{R}_{m, d}[x]$.

Consider the dynamical system

$$
\dot{x}=f(x)
$$

where $f: D \rightarrow \mathbb{R}^{n}$ is a locally Lipschitz map from the set $D \subset \mathbb{R}^{n}$ to $\mathbb{R}^{n}$. Let us assume $x=0$ is an equilibrium point, i.e. $0 \in\left\{x \in \mathbb{R}^{n} \mid f(x)=0\right\}$. Denote by $\phi(t, x(0))$ the solution to (1) that is initiated from the point $x(0)$ at time $t=0$, the set $L$ is said to be invariant with respect to (1) if $x(0)=$ $\phi(0, x(0)) \in L \Rightarrow x(t)=\phi(t, x(0)) \in L, \forall t \in \mathbb{R}$. Furthermore, $L$ is said to be positively invariant with respect to (1) if the previous implication holds for all $t \geq 0$. Given a function $R: \mathbb{R}^{n} \rightarrow \mathbb{R}$ we define the set $\mathcal{E}(R, \gamma):=\left\{x \in \mathbb{R}^{n} \mid R(x) \leq \gamma\right\}$ for some $\gamma>0$. Additionally, if it is true that $R: \mathbb{R}^{n} \rightarrow \mathbb{R}_{\geq 0}$, $\dot{R}(x)=\frac{\partial R}{\partial x} f(x) \leq 0$ for all $x \in \mathcal{E}(R, \gamma)$ then the set is contractive and invariant. We denote such sets by $\mathcal{E}_{c}(\cdot, \cdot)$. We will assume throughout this work that the function $R$ used to define any contractive set $\mathcal{E}_{c}(\cdot, \cdot)$ is a $\mathcal{C}^{1}\left(\mathbb{R}^{n}\right)$ function. The region of attraction of an asymptotically stable equilibrium point $x^{*}$ of (1) is defined as the set

$$
S:=\left\{\begin{array}{l|c}
x \in D & \phi(t, x) \text { is defined } \forall t \geq 0, \\
\lim _{t \rightarrow \infty} \phi(t, x)=x^{*}
\end{array}\right\} .
$$

The focus of this paper is to construct inner estimates of $S$. In the next section we will describe a generic algorithm form constructing an ERA.

\section{Estimating THE RA VIA LyAPUNOV LEVEL SETS}

Recall that for a locally asymptotically stable equilibrium point of (1) there must exist a Lyapunov function $V$ and a set $D \subset \mathbb{R}^{n}$ satisfying $V: D \rightarrow \mathbb{R}^{n}$ such that $V(x)>0 \forall x \in D \backslash\{0\}, V(0)=0$ and $\frac{\partial V}{\partial x} f(x)<0 \forall x \in D \backslash\{0\}$, see for example [13]. Based on this fact, a common approach to finding an ERA for systems of the form (1) is to compute Lyapunov function certifying the local asymptotic stability of the origin and obtain the largest (maximal) level set of a Lyapunov function that is contained within a set $D$ (by assumption we have $0 \in D$ ). We can describe a general algorithm based on the Lyapunov function computations to obtain ERAs in the form $\mathcal{E}_{c}(V, \gamma)$ as follows:

\section{Algorithm 1}

Step 1 (Lyapunov function computation): Given $D$, compute a Lyapunov function $V$ for $x=0$ of (1).

Step 2 (Maximization of Lyapunov level set): Given $V$ and $D$, solve

$$
\begin{array}{ll}
\operatorname{maximize} & \gamma \\
\text { subject to } & \mathcal{E}_{c}(V, \gamma) \subset D, \quad \gamma>0
\end{array}
$$

Step 3 (Update of search domain): If stopping criteria is satisfied then return ERA $\mathcal{E}_{c}(V, \gamma)$, else set new $D$, go to Step 1.

Whilst the algorithm above may look simple enough, observe that: i) In general, constructing Lyapunov functions for nonlinear dynamical systems is a non-trivial task. ii) Existing formulations for the optimization problem in Step 2 are typically nonconvex. iii) Determining how to update $D$ typically relies on some heuristic.

Step 1 asks for $\frac{\partial V}{\partial x} f(x)<0 \forall x \in D$. Except for the particular case $D=\mathcal{E}_{c}\left(V, \gamma^{*}\right)$, there is no guarantee that every $x(0) \in D$ is an initial point of a trajectory satisfying $\phi(t, x(0)) \in D \forall t>0$. Extra conditions must hold for $D$ to be an invariant set. We will expand upon this point in Section IV.

Denote the ERA and the search set from iteration $n$ of the above algorithm by $\mathcal{E}\left(V_{n}, \gamma_{n}\right)$ and $D_{n}$. Note that $D_{n} \subset D_{n+1}$ does not necessarily imply $\mathcal{E}\left(V_{n}, \gamma_{n}\right) \subset$ 
$\mathcal{E}\left(V_{n+1}, \gamma_{n+1}\right)$, which is, in general, difficult to satisfy. In fact, stopping criteria based on the increase of the ERA are seldom considered. The alternative is to consider the increase of predefined reference shape sets to evaluate the progress of the algorithm. This problem is further discussed in Section IV-B.

\section{MAin RESUlts}

Consider the system (1), where $f(x) \in \mathcal{C}^{0}\left(\mathbb{R}^{n}\right)$ is Lipschitz in $\mathcal{E}(\|x\|, r) r<R \in \mathbb{R}_{>0}$. The following results provide a set of conditions to certify that a set as $\mathcal{E}(R, r)$ is an ERA of the origin.

Theorem 1: Given a function $R: \mathbb{R}^{n} \rightarrow \mathbb{R}, R \in \mathcal{C}^{1}$ and a positive scalar $\gamma$, defining a compact set $\mathcal{E}(R, \gamma)$, if there exists a function $V: \mathbb{R}^{n} \rightarrow \mathbb{R}, V(0)=0$, $V \in \mathcal{C}^{1}$ such that

$$
\begin{gathered}
V(x)>0 \forall x \in \mathcal{E}(R, \gamma) \backslash\{0\} \\
-\langle\nabla V(x), f(x)\rangle>0 \forall x \in \mathcal{E}(R, \gamma) \\
-\langle\nabla R(x), f(x)\rangle>0 \forall x \in\left\{x \in \mathbb{R}^{n} \mid R(x)=\gamma\right\}
\end{gathered}
$$

then the solutions to the system $\dot{x}=f(x)$, $\phi\left(t, x_{0}\right)$ with $x_{0} \in \mathcal{E}(R, \gamma)$ lie in the set $S:=$ $\left\{x \in \mathbb{R}^{n} \mid \lim _{t \rightarrow \infty} \phi(t, x)=0\right\}$.

Proof: Consider the compact set $\partial \mathcal{E}(R, \gamma)$, let $\alpha=\min _{\partial \mathcal{E}(R, \gamma)} V(x)$, then from (2a), $\alpha>0$ and we have $\mathcal{E}(V, \alpha)$ satisfies $\mathcal{E}(V, \alpha) \subseteq \mathcal{E}(R, \gamma)$. From (2a), (2b) we have

$$
\begin{gathered}
V(x)>0 \forall x \in \mathcal{E}(V, \alpha) \\
-\langle\nabla V(x), f(x)\rangle>0 \forall x \in \mathcal{E}(V, \alpha) .
\end{gathered}
$$

Following [13, Theorem 4.1] we have that the origin is an asymptotic equilibrium which region of attraction is estimated by $\mathcal{E}_{c}(V, \alpha)$, that is, every trajectory $\phi\left(t, x_{0}\right)$ with $x_{0} \in \mathcal{E}(V, \alpha)$ satisfy $\phi\left(t, x_{0}\right) \rightarrow 0$ as $t \rightarrow \infty$.

Since $\mathcal{E}(R, \gamma)$ is compact and $f(x)$ is locally Lipschitz in any compact set, we have uniqueness of solutions to $\dot{x}=f(x)$, for all $x_{0} \in \mathcal{E}(R, \gamma)$, provided every solution lies entirely in $\mathcal{E}(R, \gamma)$. Assume $\exists x_{0} \in$ $\mathcal{E}(R, \gamma)$ for which the solution leaves the set, then, there must exist $T^{*}$ such that $x\left(T^{*}\right)=\phi\left(T^{*}, x_{0}\right)$ satisfies $R\left(x\left(T^{*}\right)\right) \geq 0$. From the continuity of solution and continuity of $R(x)$ there must exist $\bar{T}$, $0<\bar{T}<T^{*}$ such that $R(x(\bar{T}))=\gamma$ and $\dot{R}(x(\bar{T}))=$ $\langle\nabla R(x(\bar{T})), f(x(\bar{T}))\rangle>0$, which contradicts (3). Hence $\mathcal{E}(R, \gamma)$ is an invariant set.

Finally let us now prove that every trajectory starting in the compact set $\mathcal{E}(R, \gamma) \backslash \mathcal{E}(V, \alpha)$ enters the invariant and contractive set $\mathcal{E}_{c}(V, \alpha)$, that is that there exist a $T$ such that $\phi\left(t, x_{0}\right) \in \mathcal{E}(V, \alpha) \forall t>$ $T$. Let $\beta=\max _{\partial \mathcal{E}(R, \gamma)} V(x)$. Since (2b) holds in $\mathcal{E}(R, \gamma) \backslash \mathcal{E}(V, \alpha)$ let $-\gamma=\max _{\mathcal{E}(R, \gamma) \backslash \mathcal{E}(V, \alpha)} \dot{V}(x)$ which exists because the continuous function has a maximum over any compact set, from (2b) we also get

$$
V(x(t))=V(x(0))+\int_{0}^{t} \dot{V}(x(\tau)) d \tau \leq V(x(0))-\gamma t
$$

hence, since $V(x(0)) \leq \beta \forall t>\frac{\beta-\alpha}{\gamma}$ we have $V(x(t)) \leq \alpha$ and therefore $\phi\left(t, x_{0}\right) \in \mathcal{E}(V, \alpha)$.

Remark 1: Boundedness of the set $\mathcal{E}(R, \gamma)$ also guarantees uniqueness of solutions if the vector field is not globally Lipschitz (as for instance, the polynomial vector fields studied in section IV-B). The boundedness (actually the Archimedian property) is also required for the representation theorem (i.e. Positivstellensatz) to be applied and hence transform the conditions on Theorem 1 in SOS constraints.

Remark 2: In the case $R(x)=V(x)$, (2b) implies (2c) and compactness of the set results from the fact that $V(x)$ is strictly increasing.

Remark 3: Note that that $R(x)$ is not required to be positive define, however it is required that $\mathcal{E}(R, \gamma)$ is compact and contains the the origin. This guarantees that $\exists \alpha=\min _{\partial \mathcal{E}(R, \gamma)}(V(x))$, such that $\mathcal{E}(V, \alpha) \subseteq$ $\mathcal{E}(R, \gamma)$

The following result considers invariant regions defined by the maximum of a set of differentiable functions:

Theorem 2: Given a set of continuously differentiable functions $R_{i}: \mathbb{R}^{n} \rightarrow \mathbb{R}, i=1, \ldots, d$, defining $R_{M}(x):=\max _{i} R_{i}(x)$ and a positive scalar $\gamma$ such that the set $\mathcal{E}\left(R_{M}, \gamma\right)$ is compact, if there exists a function $V: \mathbb{R}^{n} \rightarrow \mathbb{R}_{\geq 0}, V(0)=0, V \in \mathcal{C}^{1}$ such that

$$
\begin{gathered}
V(x)>0 \forall x \in \mathcal{E}\left(R_{M}, \gamma\right) \backslash\{0\} \\
-\langle\nabla V(x), f(x)\rangle>0 \forall x \in \mathcal{E}\left(R_{M}, \gamma\right) \\
-\langle\xi, f(x)\rangle>0 \forall x \in \partial \mathcal{E}\left(R_{M}, \gamma\right), \xi \in \frac{\partial R_{M}(x)}{\partial x}
\end{gathered}
$$

where $\frac{\partial R_{M}(x)}{\partial x}$ denotes the subdifferential of $R_{M}(x)$ at $x$, then all trajectories of the system $\dot{x}=f(x)$ starting in the set $\mathcal{E}\left(R_{M}, \gamma\right)$ converge to the origin.

Remark 4: Notice that $R_{M}(x)=R_{i}(x) \forall x \in\{x \in$ $\left.\mathbb{R}^{n} \mid R_{i}(x)-R_{j}(x) \geq 0, j=1, \ldots, d\right\}$. By defining

$$
\begin{aligned}
\mathcal{M}_{i}\left(R_{M}, \gamma\right): & =\left\{x \in \mathbb{R}^{n} \mid R_{i}(x) \leq \gamma,\right. \\
& \left.R_{i}(x)-R_{j}(x) \geq 0, j=1, \ldots, d\right\} .
\end{aligned}
$$

we may write

$$
\mathcal{E}\left(R_{M}, \gamma\right)=\bigcup_{i=1}^{d} \mathcal{M}_{i}\left(R_{M}, \gamma\right)
$$

The subdifferential for the function $R_{M}(x)$ is given by $\frac{\partial R_{M}(x)}{\partial x}:=\operatorname{co}\left\{\nabla R_{\ell}(x), \ell \in \mathcal{I}(x)\right\}$, where $\mathcal{I}(x)=\left\{i \stackrel{\in}{\in}\{1, \ldots, d\} \mid R_{i}(x)=R_{M}(x)\right\}$ denotes the set of "active" functions at point $x$. Notice that $R_{M}(x)$ is not differentiable at points $x$ where $\exists i, j \in$ 
$\mathcal{I}(x), i \neq j$, that is, at points satisfying $R_{M}(x)=$ $R_{i}(x)=R_{j}(x), i \neq j$. At such points $\frac{\partial R_{M}(x)}{\partial x}$ defines a set, hence (4c) describe a set of inequalities. Whenever $\mathcal{I}(x)$ contains only one element, say $\mathcal{I}(x)=\{k\}$, $\frac{\partial R_{M}(x)}{\partial x}$ is a singleton given by $\nabla R_{k}$, which exists since $R_{i}(x) \in \mathcal{C}^{1}, \forall i=1, \ldots, d$.

The proof of Theorem 2 follows closely the proof of Theorem 1 and is therefore omitted. The only difference is related to the lack of differentiability of $R_{M}(x)$ which gives $\dot{R}_{M}(x(t)) \in\left\langle\nabla R_{M}(x(t)), f(x(t))\right\rangle$. Therefore provided (4c) holds we can use it to arrive at a contradiction as in the proof of Theorem 1 .

\section{A. Uncertain systems}

In this section we establish conditions for invariant sets to be robust estimates of the region of attraction with parameter-dependent Lyapunov functions (PDLF). Recently, PDLFs proved to be an efficient tool to certify the stability of linear system with parametric uncertainties [15]. They have also been successfully applied to obtain certificates for the local stability of polynomial systems, leading to parameterdependent estimates of the RA. A robust estimate is then obtained as the intersection of the estimates given for each fixed parameter value [4], [6].

Consider the uncertain system

$$
\dot{x}=f(x, \theta), \quad \theta \in \Theta \subset \mathbb{R}^{p}
$$

where $f: D \times \Theta \rightarrow \mathbb{R}^{n}$ and $\Theta$ denotes the uncertainty set. We assume that $f$ is sufficiently well behaved so as to provide uniqueness and local existence of solutions $^{1}$.

It is also a requirement that $f(0, \theta)=0 \forall \theta \in \Theta$. We are interested in determining a robust estimate for region of attraction (RERA) i.e. an estimate of the RAs for all dynamical systems of the form (5) with a fixed $\theta \in \Theta$ :

$S_{\theta}:=\bigcap_{\theta \in \Theta}\left\{\begin{array}{l|l}x_{0} \in D & \begin{array}{c}\phi(t, x, \theta) \text { is defined } \forall t \geq 0 \\ \lim _{t \rightarrow \infty} \phi\left(t, x_{0}, \theta\right)=x^{*}\end{array}\end{array}\right\}$

where $\phi\left(t, x_{0}, \theta\right)$ is a solution to (5) starting from $x_{0}$ at time $t$ with fixed $\theta \in \Theta$. Without loss of generality we shall assume that $x^{*}=0$ is the equilibrium of interest.

Under these assumptions that guarantee the wellposedness of system (5) the following result extends the results of Theorem 1 to the case of uncertain systems and is presented without proof.

Theorem 3: Consider the uncertain dynamical system described by (5) where $\Theta$ is a compact set and the uncertainty enters in a multiplicative manner. Given a function $R: \mathbb{R}^{n} \rightarrow \mathbb{R}, R \in \mathcal{C}^{1}$ and a positive scalar $\gamma$,

\footnotetext{
${ }^{1}$ Broadly put, this requires $f$ to be continuous in $(x, \theta, t)$ and locally Lipschitz in $x$ (uniformly in $\theta$ and $t$ ) on a bounded domain. Exact conditions can be found in any standard textbook, see for example [13, Theorem 3.5]
}

which defines the compact set $\mathcal{E}(R, \gamma)$, if there exists a function $V: \mathbb{R}^{n} \times \Theta \rightarrow \mathbb{R}, V(0, \cdot)=0, V \in \mathcal{C}^{1}$ such that

$$
\begin{gathered}
V(x, \theta)>0 \forall x \in \mathcal{E}(R, \gamma) \backslash\{0\} \times \Theta \\
-\langle\nabla V(x), f(x, \theta)\rangle>0 \forall(x, \theta) \in \mathcal{E}(R, \gamma) \times \Theta \\
-\langle\nabla R(x), f(x, \theta)\rangle>0 \\
\forall(x, \theta) \in\left\{x \in \mathbb{R}^{n} \mid R(x)=\gamma\right\} \times \Theta
\end{gathered}
$$

then the solutions to (5), $\phi\left(t, x_{0}, \theta\right)$ with $x_{0} \in \mathcal{E}(R, \gamma)$ and $\theta \in \Theta$ lie in the set $S_{\theta}$.

\section{B. Estimating the RA}

We now illustrate how Theorems 1-3 can be used in an algorithmic manner to obtain estimates for the RA. We start by presenting a generic algorithm, analogous to Algorithm 1 in Section (III), except that the obtained ERA is not given by Lyapunov level sets:

\section{Algorithm 2}

Step 1 (Invariant set enlargement): Given a function $R$ satisfying the conditions of Theorem 1, maximize $\gamma$ subject to (2).

Step 2 (Update function $R$ ): If stopping criteria is satisfied then return ERA $\mathcal{E}(R, \gamma)$; else compute an update $\widehat{R}$, make $R \leftarrow \widehat{R}$ and go to step 1 .

Later in this section we will show how it is possible to enforce the constraint that $\mathcal{E}\left(\widehat{R}, \gamma^{*}\right) \supseteq \mathcal{E}\left(R, \gamma^{*}\right)$ where $\gamma^{*}$ denotes an optimal solution to step 1 .

The following lemma will be instrumental in the rest of the paper:

Lemma 1 ( [16]): Given $h_{0}, \ldots, h_{m_{1}} \in \mathbb{R}[x]$ and $g_{1}, \ldots, g_{m_{2}} \in \mathbb{R}[x]$ with $x \in \mathbb{R}^{n}$ then the set containment

$$
\begin{aligned}
\left\{x \mid \begin{array}{r}
h_{1}(x) \\
g_{1}(x)=0, \ldots, h_{m_{1}}(x)
\end{array}\right. & \geq 0 \\
& \subseteq\left\{x \mid h_{0}(x) \geq 0\right\}
\end{aligned}
$$

holds if there exist sum of squares polynomials $s_{1}, \ldots, s_{m_{1}} \in \Sigma_{n, d_{i}}[x]$ for $d_{i}=1, \ldots, m_{1}$ and polynomials $p_{1}, \ldots, p_{m_{2}} \in \mathbb{R}_{n, d_{i}}[x]$, for $d_{i}=1, \ldots, m_{2}$ such that

$$
h_{0}(x)-\sum_{i=1}^{m_{1}} s_{i}(x) h_{i}(x)+\sum_{j=1}^{m_{2}} p_{i}(x) g_{j}(x) \in \Sigma[x] .
$$

The lemma above is a specific case of the Positivstellensatz, see [17] for further details. Note that when the degree bounds $d_{i}$ are fixed then (if feasible) the polynomials and SOS polynomials can be found using convex optimization. This process can be automated using freely available software such as SOSTOOLS [18].

It will now be shown how Algorithm 2 can be used as a basis for implementing the results from Theorem 1 when $f(x) \in \mathbb{R}^{n}[x]$, i.e. a polynomial function, and all the necessary sets are semi-algebraic. 
Applying Lemma 1 to (2a)-(2c) in Theorem 1 gives:

$$
\begin{aligned}
V(x)-m_{1}(x)(\gamma-R(x)) & \in \Sigma[x] \\
-\langle\nabla V(x), f(x)\rangle-m_{2}(x)(\gamma-R(x)) & \in \Sigma[x] \\
-\langle\nabla R(x), f(x)\rangle-p_{1}(x)(\gamma-R(x)) & \in \Sigma[x]
\end{aligned}
$$

where

$$
m_{1}, m_{2} \in \Sigma[x], \quad p_{1}(x) \in \mathbb{R}[x] .
$$

Consider also $S(x) \in \Sigma[x]$ and use Lemma 1 to formulate the set constraint

$$
\mathcal{E}\left(S, \gamma^{*}\right) \supseteq \mathcal{E}\left(R, \gamma^{*}\right)
$$

as

$$
\begin{array}{r}
(S(x)-R(x))-m_{3}(x)(\gamma-S(x)) \in \Sigma[x] \\
m_{3} \in \Sigma[x] .
\end{array}
$$

The expressions above can be used to formulate the following bilinear sum of squares programme

$$
\begin{aligned}
\underset{V, m_{i}, \gamma, R, p_{1}}{\operatorname{maximize}} & \gamma \\
\text { subject to } & (6 \mathrm{a})-(6 \mathrm{~d}),(8 \mathrm{a})-(8 \mathrm{~b}) .
\end{aligned}
$$

which is a non-convex optimization problem, that can only guarantee local optimal solutions. Any solution $\gamma^{*}$ to the above problem guarantees that the set $\mathcal{E}\left(S, \gamma^{*}\right)$ is contained in the set $\mathcal{E}\left(R, \gamma^{*}\right)$ which is an ERA as conditions in Theorem 1 hold as (6).

We observe that the problem is affine in the decision variables if either variable $R$ or variables $\left\{m_{i}, p_{1}\right\}$ are fixed in constraints (6a)-(6d), (8a)-(8b). The algorithm below explore this fact to specialize Algorithm 2 and obtain ERAs for polynomial systems through the solution of semi-definite programs.

\section{Algorithm 3}

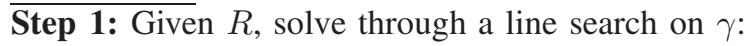

$$
\underset{V, m_{1}, m_{2}, \gamma, p_{1}}{\operatorname{maximize}} \gamma \quad \text { subject to }(6 \mathrm{a})-(6 \mathrm{~d})
$$

Step 2: Using $m_{1}, m_{2}, p_{1}$ and $\gamma^{*}$ from step 1, set $S(x)=R(x)$ and solve with a line search on $\gamma$ :

$\operatorname{maximize}_{V, R, m_{3}, \gamma} \gamma$ subject to (6a)-(6d), (8a) (8b), $\gamma \geq \gamma^{*}$.

Remark 5: Notice that the Lyapunov function is a decision variable in every step of the above algorithms. This is not the case only if one imposes $V(x)$ to be the invariant set (that is, if $R=V$ ).

Remark 6: The above algorithm guarantees a sequence of non-decreasing ERAs, however, it does not guarantee the convergence to global optimal solutions. Moreover, a function $R(x)$ satisfying (6a)-(6d) is required for its initialization. This function can be taken as any Lyapunov function satisfying the local stability of the origin.

\section{NumericAl EXAMPLE}

Consider the system $\dot{x}=f(x)$ with $f(x)$ : $\mathbb{R}^{2} \rightarrow \mathbb{R}^{2}$ given by

$$
f(x)=\left[\begin{array}{c}
-x_{1}\left(1-x_{1} x_{2}\right) \\
-x_{2}
\end{array}\right]
$$

which satisfy $f(0)=0$. Despite the fact that only the origin is an equilibrium point, its RA is not the whole of $\mathbb{R}^{n}$ nor it is bounded by a limit-cycle. The boundary of the RA is given by $\left\{x \in \mathbb{R}^{n} \mid x_{1} x_{2}=2\right\}$ (obtained from analytical solution to Zubov's equation [2, p.73]).

We apply Algorithm 3 to obtain ERA to the origin given by a function $R(x)$ of degree $\operatorname{deg}(R)=4$ while the LF satisfying the conditions for the stability satisfy $\operatorname{deg}(V)=6$. The obtained ERA after 2 iterations is presented in Figure 1. Notice that, as observed, the boundary of the estimate lies in the set where $\dot{R}<0$ but $\dot{R}<0 \forall x \in \mathcal{E}(R, \gamma)$ is not satisfied. This is not possible when strictly increasing functions as LF are used to estimate the RA. In Figure 2 for comparison we present the maximal Lyapunov set that could be obtained with the function $V$ used as a certificate for $\mathcal{E}(R, \gamma)$. Notice that with the invariant set we are able to use a LF to obtain a larger estimate than the corresponding maximal Lyapunov set (however the estimate does not contain the maximal Lyapunov set).

Remark 7: In order to guarantee the compactness of the set $\mathcal{E}(R, \gamma)$, function $R$ is required to be a positive-definite function. Which, for polynomial functions, imposes some requirements on the leading and trailing monomial degrees.

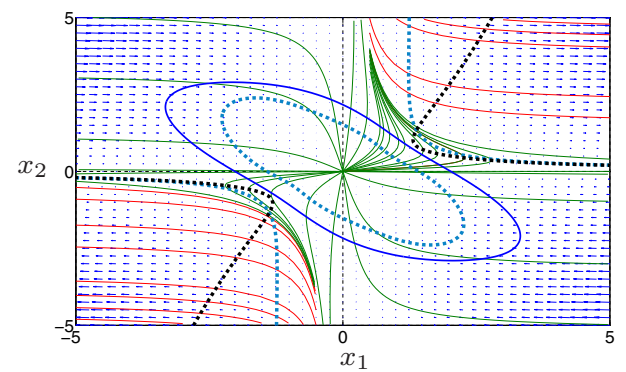

Fig. 1. The boundary of the ERA given by set $\mathcal{E}(R, \gamma)$ is depicted in dark blue while dotted light blue depicts the set $\{x \in$ $\left.\mathbb{R}^{n} \mid \dot{R}(x)=0\right\}$. The set $\left\{x \in \mathbb{R}^{n} \mid \dot{V}(x)=0\right\}$ is depicted with dotted black curve. Trajectories obtained with (V) are depicted in green (converging) and red (diverging).

In order to obtain numerical conditions for estimates with function $R_{M}(x)$, Lemma 1 is used to specialize Algorithm 2 to Theorem 2 in a similar fashion as Algorithm 3 is specified for Theorem 1. Applying the obtained algorithm to (V) gives, after the same number of iterations as in the previous case an ERA as $\mathcal{E}\left(R_{M}, \gamma\right)$ which contains the set $\mathcal{E}(R, \gamma)$. This result is depicted in Figure 3. 


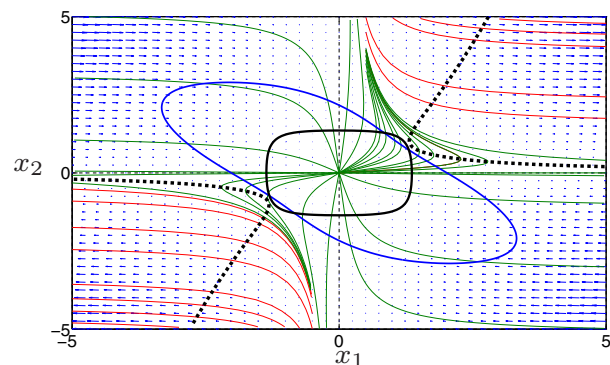

Fig. 2. Invariant ERA (in blue) and maximal Lyapunov set (in black) obtained with the LF used to certify $\mathcal{E}(R, \gamma)$.

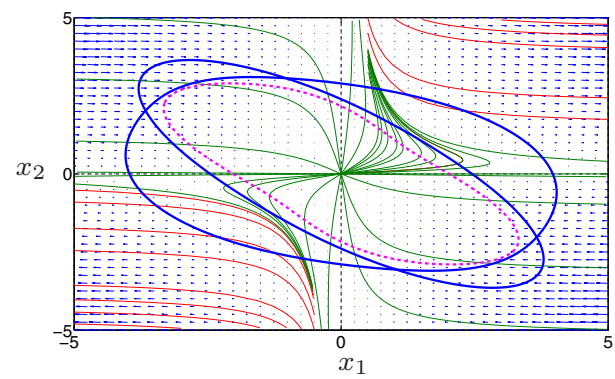

Fig. 3. Invariant ERA is given by the intersection of the sets limited by the blue curves. The set depicted in Figure 1 is given by the dashed magenta curve.

\section{CONCLUSION}

In this paper we presented conditions for an invariant set to be the ERA of the origin. The main feature of the results presented is that the ERA is associated to a Lyapunov function is an invariant set but is not given by a level set of the Lyapunov function. These results were instrumental to formulate an algorithm for the estimation of the RA that guarantees the increment of the estimate at each iteration. We the applied the algorithm to the class of polynomial vector fields and semi-algebraic sets for which the steps are performed via the solution to Sum-of-squares programs. The extension of the results to the class of systems with parametric uncertainties was also presented. For this case the invariant ERA does not depend on the uncertain parameters while the associate Lyapunov can depend on these parameters.

In [3], the concept of maximal Lyapunov functions (MLFs) was introduced. Such functions provide the complete characterization of the RA and satisfy $V(x) \rightarrow \infty$ as $x \rightarrow \partial R$. In that paper it is further shown that rational functions (given by the ratio of two polynomials) can arbitrarily approximate MLFs and also a numerical method to compute these approximations for analytical vector fields is provided. One relevant feature of those results is that the obtained ERA are described in terms of the denominator of the rational function. We are currently investigating the class of rational Lyapunov functions and pursuing the connection between the invariant analysis here presented and the conditions for stability derived with rational Lyapunov functions. We expect to provide a convex characterization in terms of a sequence of SOS programs that allows for the construction of a rational Lyapunov functions by certifying rational inequalities while computing polynomial inequalities.

\section{REFERENCES}

[1] T. C. Wang, S. Lall, and T. Y. Chiou, "Polynomial method for PLL controller optimization," Sensors, vol. 11, no. 7, pp. 6575-6592, 2011.

[2] V. I. Zubov, Methods of AM Lyapunov and their Application. Noordhoff Groningen, 1964.

[3] A. Vannelli and M. Vidyasagar, "Maximal Lyapunov functions and domains of attraction for autonomous nonlinear systems," Automatica, vol. 21, no. 1, pp. 69 - 80, 1985.

[4] G. Chesi, "On the estimation of the domain of attraction for uncertain polynomial systems via lmis," in Decision and Control, 2004. CDC. 43rd IEEE Conference on, vol. 1, 2004, pp. 881-886 Vol.1.

[5] U. Topcu and A. Packard, "Local stability analysis for uncertain nonlinear systems," IEEE Transactions on Automatic Control, vol. 54, no. 5, pp. 1042-1047, 2009.

[6] U. Topcu, A. Packard, P. Seiler, and G. J. Balas, "Robust region-of-attraction estimation," IEEE Transactions on Automatic Control, vol. 55, no. 1, pp. 137-142, 2010.

[7] W. Tan and A. Packard, "Stability region analysis using polynomial and composite polynomial Lyapunov functions and sum-of-squares programming," IEEE Transactions on Automatic Control, vol. 53, no. 2, pp. 565-571, 2008.

[8] J. B. Lasserre, Moments, Positive Polynomials and Their Applications. Imperial College Press, London, 2009.

[9] G. Chesi, "Rational Lyapunov functions for estimating and controlling the robust domain of attraction," Automatica, vol. 49, no. 4, pp. $1051-1057,2013$.

[10] G. Chesi., Domain of Attraction: Analysis and Control via SOS Programming., ser. Lecture Notes in Control and Information Sciences. Springer, 2011, no. 415.

[11] D. Henrion and J.-B. Lasserre, "Inner approximations for polynomial matrix inequalities and robust stability regions," IEEE Transactions on Automatic Control, vol. 57, no. 6, pp. 1456-1467, 2012.

[12] H.-D. Chiang, M. W. Hirsch, and F. F. Wu, "Stability regions of nonlinear autonomous dynamical systems," IEEE Transactions on Automatic Control, vol. 33, no. 1, pp. 16-27, 1988.

[13] H. K. Khalil, Nonlinear systems. Prentice hall Upper Saddle River, NJ, 2002, vol. 3.

[14] P. A. Parrilo, "Structured semidefinite programs and semialgebraic geometry methods in robustness and optimization," Ph.D. dissertation, Caltech, Pasadena, CA, 2000.

[15] R. Oliveira and P. L. Peres, "Parameter-dependent $1 \mathrm{mis}$ in robust analysis: characterization of homogeneous polynomially parameter-dependent solutions via lmi relaxations," Automatic Control, IEEE Transactions on, vol. 52, no. 7, pp. 1334-1340, 2007.

[16] Z. Jarvis-Wloszek, R. Feeley, W. Tan, K. Sun, and A. Packard, "Control applications of sum of squares programming," in Positive Polynomials in Control. Springer, 2005, pp. 3-22.

[17] G. Blekherman, P. A. Parrilo, and R. R. Thomas, Semidefinite optimization and convex algebraic geometry. SIAM, 2013.

[18] A. Papachristodoulou, J. Anderson, G. Valmorbida, S. Prajna, P. Seiler, and P. Parrilo, SOSTOOLS: Sum of squares optimization toolbox for MATLAB V3.00, Available from http: / /www.cds. caltech. edu/sostools, 2013. 